\title{
Metasurface bilayer for slow microwave surface waves
}

\author{
J. D. de Pineda $\odot,{ }^{*}$ G. P. Ward, A. P. Hibbins, and J. R. Sambles $\odot$ \\ Electromagnetic and Acoustic Materials Group, Department of Physics and Astronomy, \\ University of Exeter, Stocker Road, Devon EX4 4QL, United Kingdom
}

(Received 26 June 2019; published 23 August 2019)

\begin{abstract}
We present a simple two-layer discontinuous crossed metal-strip array that guides microwaves having very high phase and group indices. The strips are arranged on a square lattice with a two-layer unit cell. The difference in this structure resides in the length of the metal strips, which extend to several unit cells. This work focuses on the isotropic wave dispersion at the lower frequencies. In addition, two of the higher-frequency bands give rise to a very strong negative dispersion, and strong beaming occurs, which can be tailored easily by modifying the relative orientation of the layers.
\end{abstract}

DOI: 10.1103/PhysRevB.100.081409

\section{INTRODUCTION}

Metamaterials and metasurfaces have become one of the fastest developing areas in theoretical and applied electromagnetism [1]. Their proliferation as an alternative to traditional optical and microwave devices is due to their ability to provide novel control of the reflected and transmitted fields and the freedom of manipulation of guided waves that they offer. Metamaterials are artificial materials formed by periodic arrangements of subwavelength resonant elements ("meta-atoms") [2]. The resulting structures exhibit macroscopic electromagnetic or optical properties that cannot be found in nature and that go beyond those presented by the constituent materials. These properties can be tailored by simply changing the spacing, orientation, and specific features of the subwavelength elements from which they comprise [3]. At microwave frequencies, metamaterials often comprise arrays of metallic inclusions in a dielectric host environment. Metasurfaces are an effectively two-dimensional simplification of the metamaterial concept. They normally comprise a single layer or a small number of layers of meta-atoms, and are of subwavelength thickness [4].

In most cases, metasurfaces present a better alternative to bulk metamaterials for three main reasons. Bulk periodic arrays are harder and more expensive to implement, particularly when the size of the elements is reduced. Second, the operation of metamaterial devices requires the fields to propagate for several wavelengths inside the material and, thus, it is constrained by the high losses that accompany resonant elements. Due to their lower absorption and losses, metasurfaces may provide better ways to manipulate the flow of energy than bulk metamaterials [5]. Finally, metasurfaces may be extremely thin and lightweight layers that can be integrated easily into many devices. This provides a novel approach to antenna design and other microwave components, especially for space and military applications [6].

*jd602@exeter.ac.uk
Slow waves have been widely studied at optical frequencies, where line defects in two-dimensional (2D) all-dielectric photonic crystals have been used to engineer reduced group velocities [7-9]. Drude-like metals also provide slow propagation of energy associated with the excitation of surface plasmon polaritons, but these suffer from Joule losses [10]. Here, we use a bilayer metallic metasurface that supports slow modes, guided in the dielectric between the two layers. Our geometry also allows for grading of the metasurface geometry across the surface to achieve a wide range of phase velocity (or modal index) in order to make in-plane devices that reshape the surface wave fronts.

Microwave metasurfaces have shown unprecedented capability in manipulating electromagnetic waves [11], enabling unique electromagnetic phenomena such as anomalous refraction or reflection [12,13], polarization conversion [14], flat lensing [15], broadband hologram [16], electromagnetic phase shifting [17], and even propagating wave to surface wave transformations [18].

This range of manipulation of the electromagnetic fields was further increased with the introduction of transformation optics by Pendry et al. in 2006 [19]. Transformation optics represents the distortion of the electromagnetic fields as a coordinate transformation, that results in a new set of constitutive equations where the permittivity and permeability of the materials vary across the structure. This allows for traditional electromagnetic devices to be improved and reduced in size $[20,21]$. It has also played an important role in the design of new classes of imaginative devices such as electromagnetic cloaks [22]. However, for most of these devices a large range of permittivity, or mode index, is required. This introduces a new challenge in the field of metamaterials: the design of structures with a high mode index and a relatively low loss that allow for a wide range of operation frequency.

\section{METASURFACE DESIGN}

Here, we design, model, and test a very simple, ultrathin, lightweight structure that offers high index and low loss by the use of a pair of metasurface layers. The structure comprises 


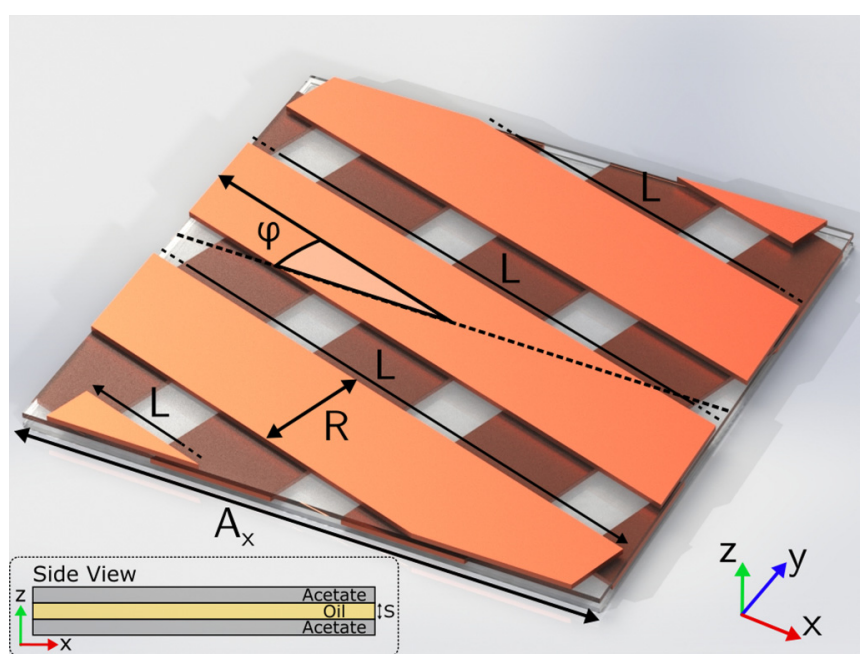

FIG. 1. Single unit cell of the structure with square lattice periodicity $A=2.5 \mathrm{~mm}$. It consists of two arrays of strips whose dimensions are length $L=7.5 \mathrm{~mm}$, width $R=0.5 \mathrm{~mm}$, and thickness $t_{\text {metal }}=18 \mu \mathrm{m}$. They are rotated by $\phi=18.43^{\circ}$ from one of the lattice axes (dashed line). The bottom layer is also rotated by $\phi=90^{\circ}$ relative to the top layer, forming a cross pattern. The copper patches are printed over a dielectric slab of permittivity $\varepsilon=2.8$ and thickness $t_{\mathrm{d}}=25 \mu \mathrm{m}$. Both these layers are joined together with an oil layer of thickness $t_{\mathrm{oil}}=15 \mu \mathrm{m}$ and permittivity $\varepsilon_{\mathrm{oil}}=3.4$. Inset: A side-on view of the unit cell that shows its three constituent layers.

two identical patterned copper layers each formed of parallel broken metal strips. The strips in the two layers are identical but orthogonal to each other. This simple geometry is shown in Fig. 1.

The difference in this structure resides in the length of the individual strips $(L=7.5 \mathrm{~mm})$ being much longer than the unit vector $(A=2.5 \mathrm{~mm})$. Hence, any effects due to the band gaps introduced by the periodicity occur at a much higher in-plane wave vector than the one corresponding to half-wave resonances of the long strips. The strips in the structure illustrated in Fig. 1 extend just over 3 unit cells. This choice also fixes their angle of rotation. In this case it is $\phi=18.43^{\circ}$ as $\tan \left(18.43^{\circ}\right)=\frac{1}{3}$. This is just one possible configurationsmaller angles will enable longer strips, which will in turn need to be made thinner, making the mode supported by the structure move further away from the light line.

The two layers of metallic patches are spaced by a dielectric layer. In this way, the electric field is strongly confined to this dielectric spacing. The spacing may be as little as tens of microns, ideally, as small as possible, leading to very high capacitive coupling between the strips in the two layers and consequentially to a low-frequency response and a high confinement of the fields. If the layers are placed very far apart they would not couple together and would support independent surface modes, different from those of the bilayer structure. In the present case, the separation between the layers is given by the dielectric substrate on which the rectangular patches are printed $\left(t_{\mathrm{d}}=25 \mu \mathrm{m}\right)$ plus an oil layer used to bind both dielectric sheets together. The oil layer has a thickness of $t_{\mathrm{oil}}=15 \mu \mathrm{m}$ and a permittivity of $\varepsilon=3.4$. These values were determined by using them as fitting parameters in a finite-element method (FEM) model [23].

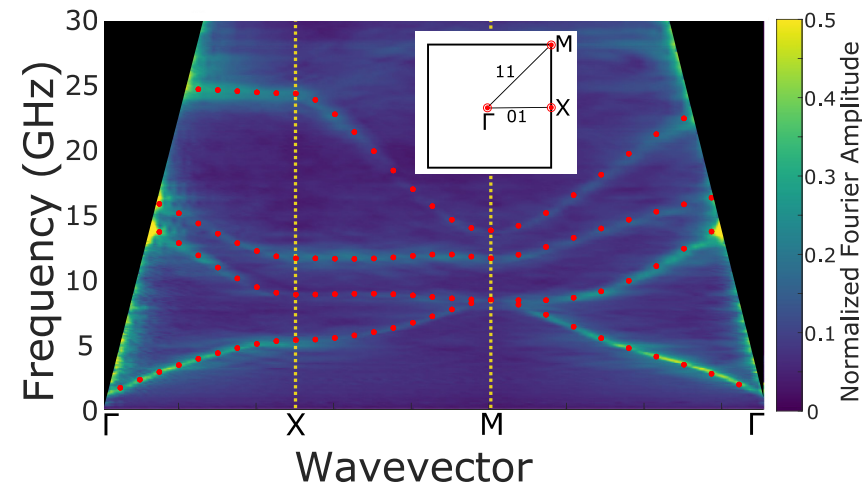

FIG. 2. Experimental dispersion of the crossed-wire sample along the three principal square lattice directions. The Brillouin zone boundaries are indicated by vertical dashed lines. The numerically modeled eigenfrequencies have been overlaid as red dots. Inset: High-symmetry points and principal square lattice directions.

\section{EXPERIMENTAL CHARACTERIZATION}

A sample was fabricated in house using a lithographic technique. The rectangular patches are printed in wax-based ink over copper-coated dielectric sheets. These are then etched with a ferric chloride solution that dissolves the exposed copper coating. Finally, the layers are aligned and bound together using the surface tension from a thin oil layer placed between them. The experimental dispersion relation of the modes supported by the structure was measured using a vector network analyzer (VNA) and two coaxial cables, each terminated with a loop antenna, whose size (radius $=1 \mathrm{~mm}$ ) is smaller than the unit cell of the structure. One of the ports on the VNA is used to excite the modes in the structure and the other one measures the amplitude and phase of the fields propagating across the structure. We use loop antennas that maximize the detection of the out-of-plane magnetic field as the electric field is strongly confined to the dielectric medium between the metallic patches while the magnetic field forms loops between adjacent patches.

The antenna used as a source is on one side of the sample and the detector antenna scans a surface area of $200 \mathrm{~mm} \times$ $200 \mathrm{~mm}$ on the opposite side. Both antennas are placed within $0.5 \mathrm{~mm}$ of the surface.

With the VNA, we record the amplitude of the electromagnetic field and its phase relative to a reference for frequencies between 0.1 and $40 \mathrm{GHz}$. By performing a spatial twodimensional Fourier transform of the surface fields at each measured frequency, the isofrequency contours in reciprocal space are obtained. From them, the dispersion diagram is plotted by taking cuts in the directions of high symmetry of the structure and stacking them together.

\section{ELECTROMAGNETIC PROPERTIES}

In Fig. 2 we illustrate the dispersion of the modes supported by the structure represented in Fig. 1 for the high-symmetry directions. The experimental measurement is compared to the dispersion predicted from a FEM numerical model (red dots), obtaining a very good agreement. To model the dispersion, we used an eigenfrequency solver. A unit cell 


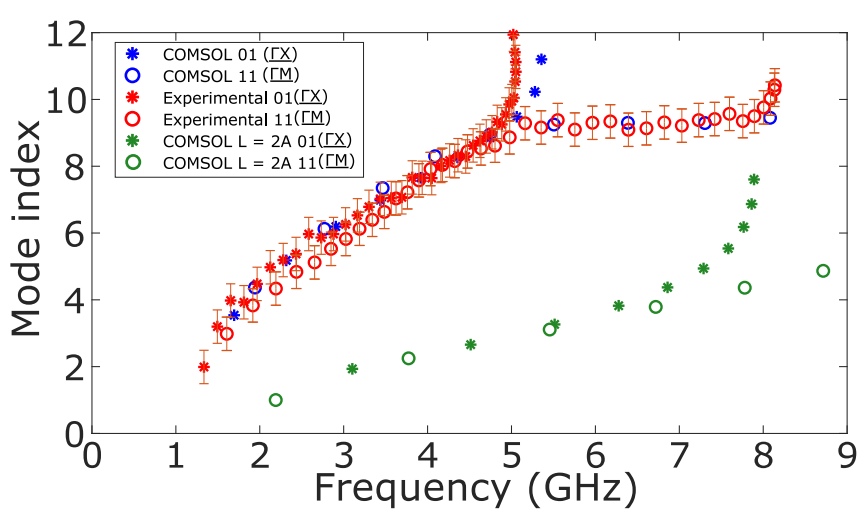

FIG. 3. Mode index of the lowest-order mode supported by the structure represented in Fig. 1. Both directions of high symmetry are represented; the (10) with stars, and (11) with circles. The mode index obtained from the experimental dispersion is represented in red with its corresponding error bars while the mode index obtained by FEM modeling is represented in blue. The mode index for strips that are just twice the length of the unit cell is shown in green.

with the parameters presented in Fig. 1 was defined, setting Floquet boundary conditions in both $x$ and $y$ directions to resemble an infinitely periodic structure.

The lowest-energy mode is very strongly dressed by the surface geometry, reducing its phase (and group) velocity significantly compared to a grazing photon (light line). This is a consequence of the patches being longer than the size of the unit cell, which is the main difference in this structure. The variation with frequency of the mode index (i.e., fractional decrease in phase velocity) for the lowest-order mode is shown in Fig. 3. In this figure the mode index calculated from FEM data (blue) is presented alongside the mode index calculated from the experimental data (red) for both the (10) and (11) directions. To calculate the mode index from the experimental data, the frequency and wave-vector values of the points of highest Fourier intensity of the mode have been taken. However, the modes have a width and this gives rise to the error bars that are also included in the plot. The isofrequency contours, that represent the modes supported by the structure for a particular frequency, have also been modeled using the FEM solver, and show very good agreement with the experimental data. Figure 4 shows these for $5 \mathrm{GHz}$. Below $5 \mathrm{GHz}$, the lowest-order mode presents almost circular isofrequency contours, meaning that the surface has an isotropic behavior. However, just above $5 \mathrm{GHz}$, the lowestorder mode reaches the Brillouin zone boundary in the (10) direction and a band gap appears, losing its isotropic behavior. For the chosen structural dimensions, the lowest-energy mode propagates near isotropically with a mode index of $\approx 8$ at frequencies around $4 \mathrm{GHz}$. However, this mode index can be easily tailored by changing the length of the strips. The dimensions presented here give rise to a high mode index which may be lowered to the dielectric spacer mode index by shortening the strips (in Fig. 3, a lower mode index is shown for strips with $L=2 A$ ). Varying the length of the strips, a high contrast graded index profile can be defined across the surface. This has a potential interest for transformation optics applications in the design of planar microwave devices.

The higher-order modes present different features such as negative dispersion (e.g., the third mode between $\Gamma$ and $X$ in Fig. 2) and strong beaming [24] in a direction which is directly related to the angle of rotation of the strips (e.g., the secondorder mode presents square-shaped isofrequency contours at $10 \mathrm{GHz}$ ). These properties are not unique to this metasurface structure and are hence not the focus of this study, but they can be tailored by changing the relative angle between the arrays.

Losses are important when designing a microwave device. The modes supported by the structure need to be strong enough throughout the entire device to ensure an acceptable performance. The losses of this structure have been quantified for the range of frequencies where the structure supports a near isotropic propagating mode (between 2 and $5 \mathrm{GHz}$ ). To quantify the losses, the time-averaged field magnitude was fitted by a function that includes a term compensating for the radial dependence and an exponential decay. From that exponential decay, we obtain the propagation length. The
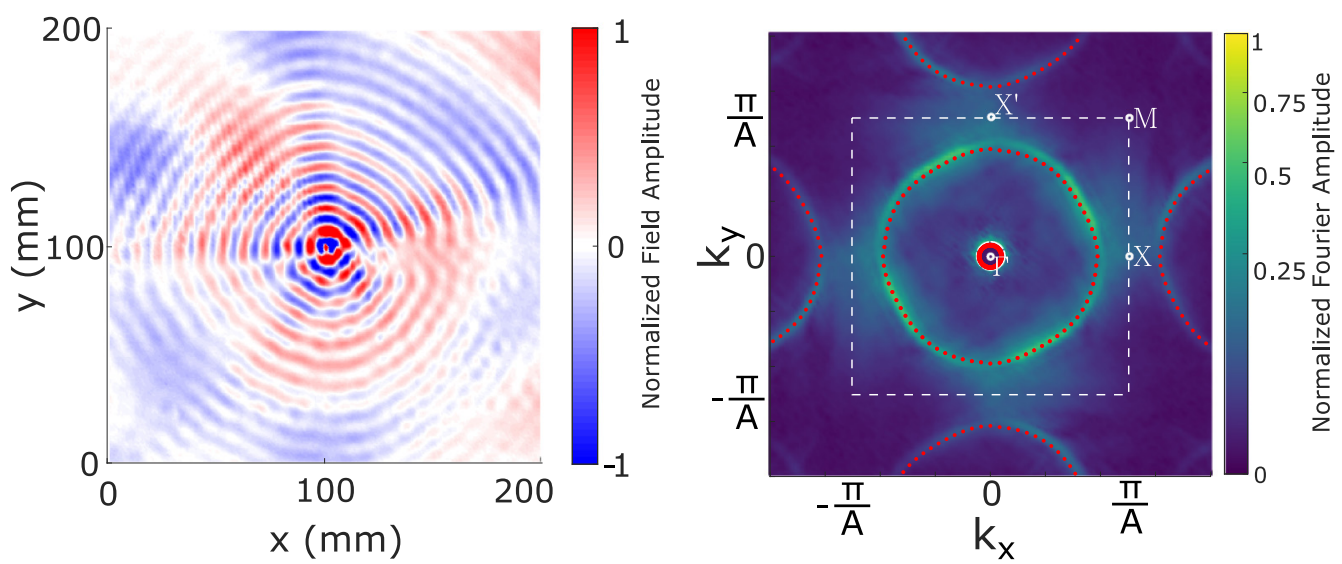

FIG. 4. Left: Experimentally measured out-of-plane component of the magnetic field at a distance of $0.5 \mathrm{~mm}$ from the surface at $5 \mathrm{GHz}$. Right: Reciprocal space map produced by a 2D Fourier transform of the electromagnetic fields represented on the left; a square root function has been applied to the data to increase the contrast. The numerically calculated eigenfrequencies are overlaid as dotted red points and the first Brillouin zone boundary is shown by dashed white lines. 
losses have also been modeled using a FEM software, taking into account the conductivity of copper and the tangent loss of the dielectric. In both cases, the propagation constant was found to be of the order of $0.01 \mathrm{~mm}^{-1}$, meaning that the fields can propagate between 100 and $200 \mathrm{~mm}$ before decaying by a factor of $e$. This is enough for optimal performance of most planar microwave devices.

\section{CONCLUSIONS}

A thin, lightweight, bilayer metasurface structure that presents the potential for guiding of surface waves in microwave applications has been presented. It is formed of metal strips much longer than the size of the unit cell. This reduces the degrees of surface anisotropy of the mode induced by band-gap effects associated with the structure's periodicity. Also, it ensures that the frequency of operation is far away from the resonant frequency of the constituent metallic elements, hence minimizing the propagation losses.

All data created during this research are openly available from the University of Exeter's institutional repository [25].

\section{ACKNOWLEDGMENTS}

We had financial support from EPSRC CDT (Grant No. EP/L015331/1) and the "Synthesizing 3D Metamaterials for $\mathrm{RF}$, microwave and $\mathrm{THz}$ applications" project (Grant No. EP/N010493/1). J.P.G. wishes to thank Flann Microwave Ltd. (Bodmin, U.K.) for additional financial support.
[1] S. B. Glybovski, S. A. Tretyakov, P. A. Belov, Y. S. Kivshar, and C. R. Simovski, Phys. Rep. 634, 1 (2016).

[2] Theory and Phenomena of Metamaterials (Metamaterials Handbook), edited by F. Capolino (CRC Press/Taylor and Francis, Boca Raton, FL, 2009).

[3] R. Liu, Q. Cheng, J. Y. Chin, J. J. Mock, T. J. Cui, and D. R. Smith, Opt. Express 17, 21030 (2009).

[4] H.-T. Chen, A. J. Taylor, and N. Yu, Rep. Prog. Phys. 79, 076401 (2016).

[5] Y. Zhao and A. Alù, Phys. Rev. B 84, 205428 (2011).

[6] G. Minatti, M. Faenzi, E. Martini, F. Caminita, P. De Vita, D. Gonzalez-Ovejero, M. Sabbadini, and S. Maci, IEEE Trans. Antennas Propag. 63, 1288 (2015).

[7] T. Baba, Nat. Photon. 2, 465 (2008).

[8] F. Wang, J. S. Jensen, J. Mørk, and O. Sigmund, J. Opt. Soc. Am. A 29, 2657 (2012).

[9] J. Li, T. P. White, L. O'Faolain, A. Gomez-Iglesias, and T. F. Krauss, Opt. Express 16, 6227 (2008).

[10] J. Villatoro, V. P. Minkovich, and J. Zubia, IEEE Photon. Technol. Lett. 27, 1181 (2015).

[11] S. Maci, G. Minatti, M. Casaletti, and M. Bosiljevac, IEEE Antennas Wireless Propag. Lett. 10, 1499 (2011).

[12] N. Yu, P. Genevet, M. A. Kats, F. Aieta, J.-P. Tetienne, F. Capasso, and Z. Gaburro, Science 334, 333 (2011).

[13] C. Pfeiffer and A. Grbic, Phys. Rev. Lett. 110, 197401 (2013).
[14] C. Pfeiffer and A. Grbic, Phys. Rev. Appl. 2, 044011 (2014).

[15] J. D. de Pineda, R. C. Mitchell-Thomas, A. P. Hibbins, and J. R. Sambles, Appl. Phys. Lett. 111, 211603 (2017).

[16] B. H. Fong, J. S. Colburn, J. J. Ottusch, J. L. Visher, and D. F. Sievenpiper, IEEE Trans. Antennas Propag. 58, 3212 (2010).

[17] R. Blanchard, G. Aoust, P. Genevet, N. Yu, M. A. Kats, Z. Gaburro, and F. Capasso, Phys. Rev. B 85, 155457 (2012).

[18] S. Sun, Q. He, S. Xiao, Q. Xu, X. Li, and L. Zhou, Nat. Mater. 11, 426 (2012).

[19] J. B. Pendry, D. Schurig, and D. R. Smith, Science 312, 1780 (2006).

[20] N. Kundtz and D. R. Smith, Nat. Mater. 9, 129 (2010).

[21] J. B. Pendry, A. Aubry, D. R. Smith, and S. A. Maier, Science 337, 549 (2012).

[22] R. Liu, C. Ji, J. J. Mock, J. Y. Chin, T. J. Cui, and D. R. Smith, Science 323, 366 (2009).

[23] J. D. de Pineda, A. P. Hibbins, and J. R. Sambles, Phys. Rev. B 98, 205426 (2018).

[24] S.-H. Kim, T.-T. Kim, S. S. Oh, J.-E. Kim, H. Y. Park, and C.-S. Kee, Phys. Rev. B 83, 165109 (2011).

[25] J. D. de Pineda, G. P. Ward, A. P. Hibbins, and J. R. Sambles, "Metasurface bilayer for slow microwave surface waves", https: //ore.exeter.ac.uk/repository/handle/10871/38406 (2019). 